\title{
Attendance for ischaemic stroke before and during COVID-19 lockdown in Singapore
}

\section{Dear Editor,}

The coronavirus disease 2019 (COVID-19) outbreak has impacted healthcare systems worldwide. Globally, visits to the emergency department have fallen as much as 25\% during COVID-19-related lockdowns. ${ }^{1}$ Notably, there have been reports that patients with acute emergencies such as strokes and heart attacks are either not seeking treatment, ${ }^{2,3}$ or are delaying treatment. ${ }^{4}$

Our study aimed to ascertain the impact of the COVID-19 pandemic and the associated lockdown restrictions on acute ischaemic stroke admissions in Singapore, using our hospital's Acute Stroke Ischaemic Audit Database, which is a database of all ischaemic stroke patients who are admitted to the Department of Neurology, at the Singapore General Hospital.

Singapore saw its first COVID-19 case on 23 January 2020. On 7 April 2020, non-essential business, workplaces and social activities were suspended, alongside a transition to home-based learning on 8 April 2020. The lockdown restrictions were eased on 2 June 2020.

We compared patients with acute ischaemic stroke who presented between 1 January 2020 and 31 March 2020 with the similar period in 2019 to ascertain differences in pre-lockdown attendances. We also made comparisons for the period of 1 April 2020 and 1 June 2020 with the similar period in 2019 to ascertain differences in lockdown attendances. Data on demographics, time from onset to arrival at hospital, stroke classification by the Oxfordshire Community Stroke Project (OCSP) $)^{5}$ classification, neurological deficit severity measured using the National Institute of Health Stroke Scale (NIHSS), ${ }^{6}$ stroke hyperacute treatment, as well as pre-admission and discharge functional status assessed using the modified Rankin scale $(\mathrm{mRS})^{7}$ were collated from the database.

Throughout the entire lockdown period, the Emergency Department in our hospital remained open with no diversion of acute ambulance services. Stroke services were not curtailed, and hyperacute therapy with both thrombolysis and endovascular therapy continued. The only change in practice was the involvement of infectious diseases triage doctors to determine risk status of patients before endovascular therapy. Acute stroke unit care continued even in isolation facilities for patients who were COVID-19 positive, or who were pending COVID-19 status. ${ }^{8}$

Statistical tests employed included the t-test, MannWhitney U test, or chi-square test as appropriate. A two-tailed alpha of 0.05 was deemed significant. All analyses were done on Stata version 16 (StataCorp LP, College Station, US).

Pre-lockdown trends. From 1 January to 31 March 2020, 234 ischaemic stroke patients (2.6 patients/ day) were admitted to our hospital, relatively similar to the 241 ischaemic stroke patients ( 2.7 patients/day) admitted in the corresponding period in 2019. While there was a trend of slightly older patients presenting in 2020 (mean of $68 \pm 12$ versus $66 \pm 13, P<0.08$ ) compared to 2019 , there was no significant differences in other demographics, stroke classification, stroke severity, treatment and functional status (Table 1). Analyses that were restricted to the months of February and March (given that the first case of COVID-19 was on 23 January 2020) showed similar results (data not shown here).

Lockdown trends. From 1 April to 1 June 2020, a total of 128 patients ( 2.1 patients/day) were admitted to our hospital, significantly fewer than the 178 patients (2.9 patients/day) who were admitted between 1 April and 1 June $2019(P=0.005)$. Patients who presented in 2020 were more likely to have large territory strokes on the OCSP scale compared with those admitted in 2019 $(P=0.007)$ (Table 1$)$. There were no other significant differences in demographic or stroke-related measures during the lockdown period.

Our study shows that cases of COVID-19 in Singapore alone did not affect stroke presentations to our tertiary stroke centre, but the implementation of a lockdown was associated with fewer stroke presentations. Individuals who presented to our tertiary stroke centre were also more likely to have large territory strokes. However, we did not detect any significant delays in presentation, nor were there any differences in the rates of tissue plasminogen activator administration when comparing the relevant periods in 2019 and 2020.

A retrospective study based in Hong Kong ${ }^{9}$ showed that during the early containment phase of COVID-19, 
Table 1. Comparison of stroke attendances between January-March 2020 versus 2019; and April-May 2020 versus 2019

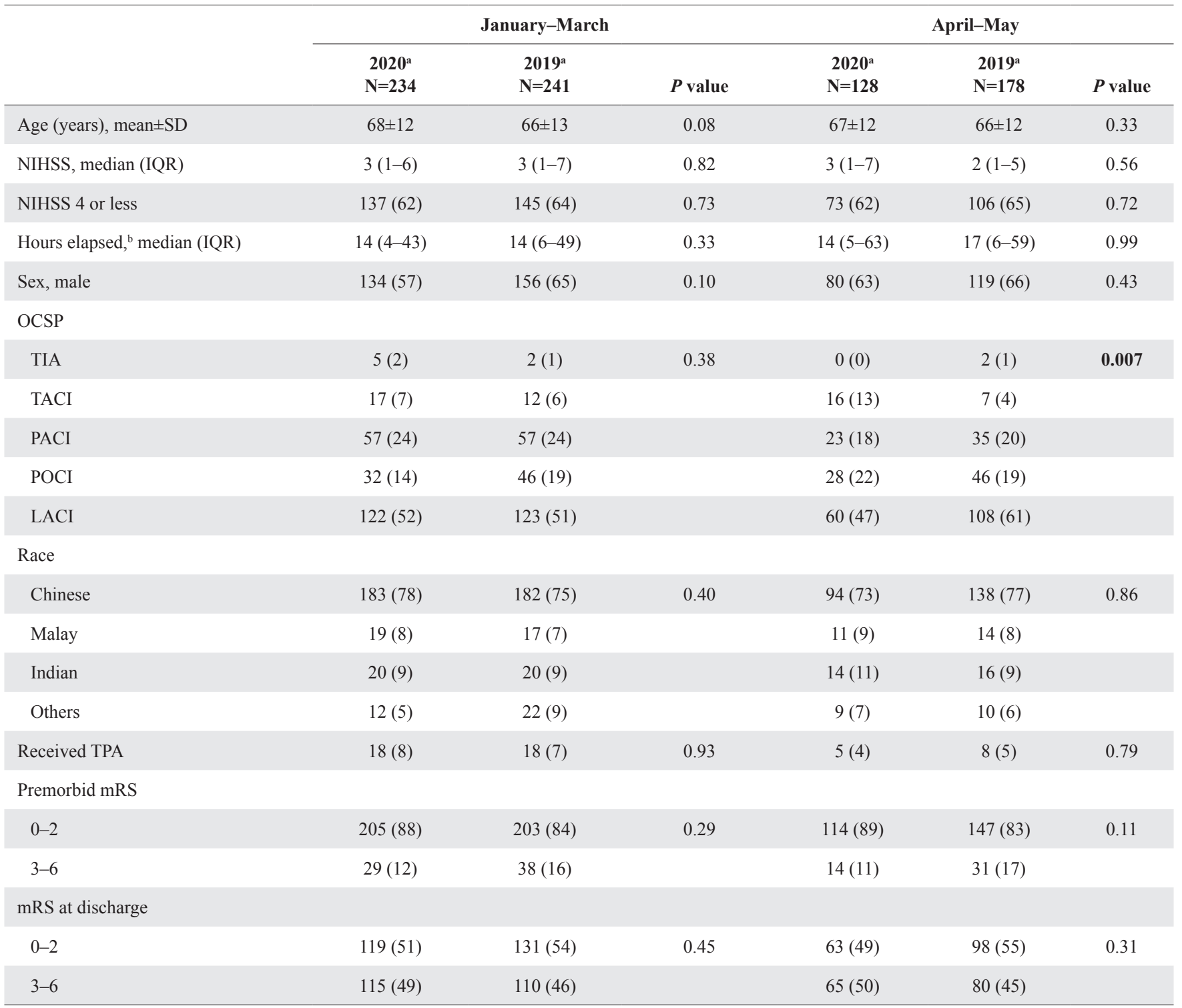

IQR: interquartile range; LACI: lacunar infarct; mRS: modified Rankin scale; NIHSS: National Institute of Health Stroke Scale; OCSP: Oxfordshire Community Stroke Project classification; PACI: partial anterior circulation infarct; POCI: posterior circulation infarct; SD: standard deviation; TACI: total anterior circulation infarct; TIA: transient ischaemic attack; TPA: tissue plasminogen activator

${ }^{a}$ Data presented as n (\%), except age, which is presented as mean \pm SD, and NIHSS and hours elapsed, which are presented as median (IQR).

${ }^{\mathrm{b}}$ Hours elapsed since symptom onset and presentation to the Emergency Department.

there was a prolongation in stroke onset to hospital arrival time, and a significant reduction in individuals arriving at hospital within the 4.5-hour window. Our study did not show a similar prolongation in stroke onset to hospital arrival time, possibly due to the presence of only 1 national ambulance service, and the relatively small land area of Singapore.

We found that there were fewer patients presenting during the lockdown period, similar to findings from an early Italian study, ${ }^{3}$ a review on the use of stroke imaging in the US, ${ }^{10}$ and a tertiary stroke centre in New Haven,
US. ${ }^{11}$ We postulate that this decline in patients presenting to the hospital is likely due to fear of coming to a hospital during a pandemic, following possible concerns about exposure to COVID-19. This may explain why lacunar strokes and transient ischaemic attacks appear to present less during the lockdown compared to large territory strokes, which are likely to affect function severe enough to warrant hospital care in the acute setting. A similar reduction in ischaemic stroke presentation was noted during the severe acute respiratory syndrome outbreak in Singapore in 2002. ${ }^{12}$ 
Our study is limited by its retrospective nature, its sole dependence on data from our stroke audit database, and the limitation of the data to a single tertiary stroke centre. As the audit database relies on inpatient admissions, we were not able to capture information on patients with possible transient ischaemic attacks who were discharged from the Emergency Department. We were unable to confirm individual patient's reasons for delays in presentation and to ascertain how many people considered COVID-19 in their decisions to present to the hospital. We were also not able to ascertain the actual number of patients who had minor strokes or transient ischaemic attacks and did not actually present to the hospital, nor those who chose to present to a different hospital. Further studies focusing on the patient's beliefs and choices in deciding when to seek treatment at a hospital during a pandemic should be explored.

Nevertheless, our study demonstrates that lockdown did impact stroke presentations to our tertiary stroke centre. There may be a role for public education to emphasise the timeliness and availability of hyperacute stroke treatment even during pandemics in order to prevent stroke patients from staying at home untreated.

\section{REFERENCES}

1. Thornton J. Covid-19: A\&E visits in England fall by $25 \%$ in week after lockdown. BMJ 2020;369:m1401.

2. Siegler JE, Heslin ME, Thau L, et al. Falling stroke rates during COVID-19 pandemic at a comprehensive stroke center. J Stroke Cerebrovasc Dis 2020;29:104953.

3. Morelli N, Rota E, Terracciano C, et al. The Baffling Case of Ischemic Stroke Disappearance from the Casualty Department in the COVID-19 Era. Eur Neurol 2020;83:213-5.

4. The Guardian. Concern as heart attack and stroke patients delay seeking help, 16 April 2020. Available at: https://www.theguardian.com/ world/2020/apr/16/coronavirus-concern-heart-attack-stroke-patientsdelay-seeking-help. Accessed on 31 August 2020.

5. Lovett JK, Coull AJ, Rothwell PM. Early risk of recurrence by subtype of ischemic stroke in population-based incidence studies. Neurology 2004;62:569-73.

6. Lyden P, Raman R, Liu L, et al. National institutes of health stroke scale certification is reliable across multiple venues. Stroke 2009;40:2507-11.

7. Bonita R, Beaglehole R. Recovery of motor function after stroke. Stroke 1988;19:1497-500.

8. De Silva DA, Tan IF, Thilarajah S. A protocol for acute stroke unit care during the COVID-19 pandemic. J Stroke Cerebrovasc Dis 2020;29:105009.

9. Teo KC, Leung WCY, Wong YK, et al. Delays in Stroke Onset to Hospital Arrival Time during COVID-19. Stroke 2020;51:2228-31.

10. Kansagra AP, Goyal MS, Hamilton S, et al. Collateral Effect of Covid-19 on Stroke Evaluation in the United States. N Engl J Med 2020;383:400-1.

11. Jasne AS, Chojecka P, Maran I, et al. Stroke Code Presentations, Interventions, and Outcomes before and during the COVID-19 Pandemic. Stroke 2020;51:2664-73.

12. Riandini T, Tan KB, De Silva DA. Lessons from Severe Acute Respiratory Syndrome Coronavirus 2003 Pandemic as Evidence to Advocate for Stroke Public Education During the Current Coronavirus Disease 2019 Pandemic. Ann Acad Med Singap 2020;49:538-42.

Kaavya Narasimhalu, ${ }^{1} M R C P$, Fung Peng Woon, ${ }^{2} M S c$, Szu Chyi Ng, ${ }_{B S C}$, Deidre Anne De Silva, ${ }^{1}{ }_{M R C P}$

\footnotetext{
${ }^{1}$ Department of Neurology, National Neuroscience Institute, Singapore General Hospital Campus, Singapore

${ }^{2}$ Department of Neurology, Singapore General Hospital, Singapore
}

Correspondence: Dr Kaavya Narasimhalu, Department of Neurology, National Neuroscience Institute (Singapore General Hospital Campus), Outram Road, Singapore 169608.

Email:nkaavya@gmail.com 